\title{
Instalaciones y nuevas formas expresivas en el arte chileno
}

\author{
Carmen Adams Fernández \\ Universidad de Oviedo
}

\begin{abstract}
RESUMEN. Dentro de las últimas tendencias del arte chileno actual la opción pictórica continúa teniendo gran relevancia, lo que se explica por la pervivencia de una tradición, pero también por la atracción que para los más jóvenes supone la personalidad y relevancia internacional de Roberto Matta. No obstante, y pese a que esta preponderancia de la pintura sobre lienzo es un hecho, también lo es la existencia de artistas que han elegido otros medios de expresión que van desde la escultura hacia esos otros lenguajes más indefinibles dentro de la clasificación tradicional de las artes, como son las instalaciones o esas realizaciones que se incluyen dentro de ese cajón de sastre denominado Arte Visual.

Palabras clave: Chile, instalaciones, sur, vanguardia

ABSTRACT. Within the latest tendencies in Chilean art, painting still bears great relevance, a fact which is partly due to the maintenace of an established tradition, but also to the attraction which the personality and international relevance of Roberto Matta holds for the youngest. However, and despite the ruling of painting on canvas, there are also a number of artists who have chosen different means of self-expression, ranging from sculpture to other languages which seem difficult to define in traditional art classification, such as installations or the realisation included within the general category of Visual Art.

Key words: Chile, Installation, South, Avant-garde.
\end{abstract}

Cuando El poeta chileno Vicente Huidobro afirma, con muy buen criterio, que los cuatro puntos cardinales son dos: norte y sur. $\mathrm{Y}$ esto ocurre además en ese orden. El norte se ubica arriba en los mapas y el sur debajo. Y es sobre este sur acerca de lo que quiero hablar aquí. Si atendemos a publicaciones de prestigio dedicadas al estudio del arte actual, encontramos como las referencias a Hispanoamérica son escasas. Así por ejemplo en el libro Arte para el siglo XXI ${ }^{1}$, editado por Burkhard Riemschneider Uta Grosenick, las alusiones a América del centro o sur son prácticamente inexistentes. Sí existen monografías dispersas, pero escasean los análisis de conjunto, los estudios generales sobre la última producción artística de esos países.

En realidad lo que aquí pretendo es reivindicar el valor de vanguardia y creación de la producción de los artistas que

1 BURKHARD RIEMSCHNEIDER UTA GROSENICK, Arte para el siglo XXI, Taschen, Italia, 1999.
} 
han venido trabajando en las últimas décadas en Chile --ese país al sur del sur--, escogiendo lenguajes al margen de la clasificación tradicional de las artes.

Es cierto que gracias a los estudios teóricos de Riegl, desde finales del XIX se ha llegado a una división más racional de las artes, frente a la tradicional de Artes mayores y menores, diferenciando arquitectura, plástica y ornamento.

Pero tampoco es que esta división sea, ni mucho menos, perfecta. Hay que tener en cuenta que la técnica, la función y la expresión de la obra no se pueden reducir exclusivamente a las categorías constructiva, figurativa o plástica y a la ornamental. Hay que considerar, además, que materiales, soportes, técnicas... han sufrido importantísimas variaciones en los últimos años. Se han llegado incluso a utilizar medios de impresión, de proyección y de generación de formas antes desconocidas.

Y es que es preciso considerar junto a las técnicas artísticas tradicionales otras más actuales, como los sistemas de impresión, proyección o generación, tanto de sonido como de imagen ${ }^{2}$.

Las clasificaciones que se han hecho respecto a las artes son en todo caso diversas. Otras que existen son las que distinguen: artes visuales o auditivas (relativas a los sentidos considerados superiores), frente a las artes relacionadas con el gusto, el tacto o el olfato. Estos tres últimos sentidos --gusto, tacto, olfato-- se consideran de esta forma incapaces de percibir formas estéticas. Es decir, se niega a las formas y objetos táctiles, gustativos y olfativos el carácter artístico. Pero esto resulta falaz, ya que recuperando para las artes estos sentidos, podemos incluir en el mundo de las artes el

2 J.Fernández Arenas, Introducción a la conservación de patrimonio y técnicas artísticas, Ariel, Barcelona, 1996.
}

arte efímero, como las obras que incluyen alimentos perecederos. También la escultura para ciegos, táctil. O pensar en exposiciones de perfumes, o en la inclusión de éstos en algunas muestras.

Dentro de las últimas tendencias del arte chileno actual la opción pictórica continúa teniendo gran relevancia, lo que se explica por la pervivencia de una tradición, pero también por la atracción que para los más jóvenes supone la personalidad y relevancia internacional de Roberto Matta. No obstante, y pese a que esta preponderancia de la pintura sobre lienzo es un hecho, también lo es la existencia de artistas que han elegido otros medios de expresión que van desde la escultura hacia esos otros lenguajes más indefinibles dentro de la clasificación tradicional de las artes, como son las instalaciones o esas realizaciones que se incluyen dentro de ese cajón de sastre denominado Arte Visual.

Para entender la evolución del arte chileno en las últimas décadas ${ }^{3}$ es destacable la primera exposición del Grupo Rectángulo en 1956 --que a mediados de los años 60 pasa a denominarse Forma y Espacio-- , y que de hecho será uno de los primeros colectivos organizados de artistas no figurativos en el país andino. Su propuesta, que lidera Ramón Vergara, es la abstracción geométrica. No obstante sus integrantes acabarán más tarde volviendo a una cierta figuración tan del gusto de la tradición pictórica chilena, pero sin perder el carácter geométrico y abstraizante de los inicios.

Más éxito tendrá el Informalismo, que llega a Chile a través de influencia es-

\footnotetext{
3 Ver además: D. BAYÓN, Artistas contemporáneos de América Latina, UNESCO, Barcelona, 1981; D. BAYÓN, Aventura plástica de Hispanoamérica, Fondo de Cultura Económica, México, 1974; V. CARVACHO HERERA, Veinte pintores contemporáneos de Chile, Santiago, 1978; S. NEGRI, Al sur del Sur, Buenos Aires, 1993; S. NEGRI, Al sur del sur, Buenos Aires, 1994
} 
pañola, en concreto de Tápies, y que arraiga con mucha más fuerza que el geometrismo intelectual de Rectángulo ${ }^{4}$. Vemos así la llegada al país andino de los planteamientos más vanguardistas por directa influencia hispana. La introducción de esta tendencia está directamente relacionada con la constitución en 1961 del Grupo Signo, integrado por Gracia Barrios, José Balmés, Eduardo Menéndez Bonati y Alberto Pérez.

Hay que considerar también el apogeo del muralismo y la denuncia durante la década de los sesenta, y sobre todo el brutal corte que supuso el golpe militar de 1973 para toda la actividad cultural chilena. Se implanta la censura, se veta a profesores, los rectores de las universidades pasan a ser militares, el toque de queda impide tertulias y funciones teatrales, lo que conllevará una auténtica parálisis del circuito artístico, y el consiguiente surgimiento de posturas estéticas autocríticas, pesimistas y desesperadas $^{5}$.

Además, Labowitz destaca cómo el arte conceptual, que aparece en Chile en la década de los 70, y que se desenvuelve con cierto retraso respecto a otros países, alcanzará un peculiar auge en tiempos de la Dictadura en su vertiente más simbólica y fácilmente interpretable. Así, señala por ejemplo que "no había manera de equivocarse sobre el significado de un grabado que en forma serial mostrara una gran cantidad de anónimas y casi idénticas fotografías de documentos de identificación, manchadas o rayadas por el color rojo-sangre".

Serán bastantes los artistas que durante la primera década de la Dictadura pierdan la confianza en el circuito artístico,

${ }^{4} \mathrm{P}$. LABOWITZ, "Apuntes sobre el arte moderno en Chile" , Artistas chilenos en España, Madrid, 1996.

${ }^{5}$ M. IVELIC, "Chile", Arte latinoamericano del siglo XX, Nerea, Madrid, 1996. tradición incompatible con la situación de inseguridad e incertidumbre en que vivían. Se produce entonces una renuncia a los lenguajes artísticos tradicionales y se buscan nuevos medios de expresión ${ }^{6}$. Así, por ejemplo el propio cuerpo servirá como soporte de significación autobiográfica ${ }^{7}$. Estos artistas reinterpretarán el mundo cotidiano: la ciudad y buscarán nuevas maneras de exponer y de circulación de obras.

Surgen a partir de ahí propuestas diversas, no concebidas como un todo con carácter de globalidad. Son experimentos que se desvinculan de los medios tradicionales y que buscan en la neovanguardia internacional formas diferentes de expresión que toman como recurso las instalaciones, la combinación interactiva de las realizaciones que surgen gracias a las nuevas tecnologías (fotografía, video..). Esto, de todas formas, convivirá como ya señalamos con un importante desarrollo de la pintura, personificada por autores de la talla de Roberto Matta o Claudio Bravo; ninguno de los cuales, por cierto, acabará trabajando en su país de origen.

Es preciso además considerar que las posibilidades económicas del arte chileno son limitadas, y que por tanto el recurrir a las nuevas tecnologías será más una utopía que una realidad. Igualmente, las instalaciones partirán muchas veces de materiales de desecho, y sus dimensiones se verán reducidas. De todas formas hay ejemplos de esta opción estética como los casos de Francisco Brugnoli, Lotty Rosenfeld y otros.

En todo caso no debemos dejar de lado la característica más importante del arte actual, chileno o no, y es su internacionalismo, ya que los nuevos medios de co-

\footnotetext{
${ }^{6}$ M. IVELIC, Opus. Cit., 1996

${ }^{7}$ Cuerpos pintados: cuarenta y cinco pintores chilenos, Cochrane, 1991; Have you seen sculpture from the body?, The Tate Gallery, Londres, 1984
} 
municación han acabado con las fronteras. Esto, por supuesto en lo que a modelos se refiere, porque en cuanto a medios está claro que no, y es lo que obliga a los artistas de países del Tercer Mundo a exiliarse, además de los eventuales motivos políticos que puedan intervenir.

Dentro de la generación de artistas conceptuales cuya obra, con un fuerte contenido ideológico, se propone denunciar los efectos de la Dictadura, encontramos a Catalina Parra. Sus instalaciones muestran la censura y la constante violación de los derechos humanos durante el régimen militar. Para ello, y por medios diversos, Parra contrapone los símbolos del poder establecido, la cultura oficial, con las manifestaciones de las minorías étnicas y sociales. Sus "reconstrucciones" son auténticos fotomontajes a partir de material impreso. Su producción puede dividirse en tres periodos que corresponden a su estancia en Alemania (1968 a 1972), Chile (1972 a 1980) y Nueva York, donde reside a partir de 1980 .

Francisco Brugnoli (n.1935) estudió en la Escuela de Bellas Artes de la Universidad de Chile y en Estados Unidos. A lo largo de su carrera simultanea su labor docente en la Facultad de Artes y en la de Arquitectura y Urbanismo de la Universidad de Chile con su vocación artística. Si bien la formación recibida durante sus estudios superiores se orientó hacia la pintura, su obra ya desde el comienzo (mediados de los años 60) presenta rasgos que exceden este campo y que incluso la ponen en tensión con él. Así sus propuestas incorporaban objetos diversos, fotografía, fragmentos de textos.

Concibe Brugnoli el objeto artístico como testigo de un pasado que puede recuperarse: son objetos usados, desechados, recogidos y atesorados, como un signo de marginalidad. La obra de este artista se sitúa entre los primeros trabajos experimen- tales y críticos con la hegemonía pictórica del panorama plástico chileno.

Son destacables sus instalaciones a partir de restos y basuras que engalana con polvo de oro, ilumina con bombillas y artificios, en un retrato amargo de la realidad iberoamericana actual. Sus obras son efímeras más por razones de índole económica que por voluntad impresionista, y esto es común a otros artistas hispanoamericanos cuya relación con propuestas de vanguardias se han de quedar en apenas acercamiento a los inaccesibles avances tecnológicos. Aquí no hay video ni laser, como mucho las bombillas de baja tensión de Brugnoli.

En este mismo escenario encontramos a Nancy Gewolb, Loty Rosenfeld o Ángela Riesco. Nancy Gewolb (n.1939) estudió Bellas Artes en la Universidad de Chile y en sus inicios presenta una provocativa pintura a base de rojos intensos y desnudos femeninos. En 1973 renuncia a la policromía y utiliza la tinta china para buscar en su propia memoria escenas de su infancia. Pero, lo más interesante de su producción serán sus composiciones tridimensionales por medio de sábanas, papeles, graffiti e iluminación, creando espacios que envuelven al espectador.

Loty Rosenfeld (n.1943 o 47) funda en 1979 el Colectivo de Acciones de Arte (CADA) junto a J.Castillo, D. Elttit y R.Zurita. Su actividad, tanto dentro del colectivo como la realizada individualmente, es una feroz crítica hacia el poder, hacia la cotidianeidad establecida, hacia la pasividad del ser humano y del artista, con un total rechazo respecto a los espacios habituales de difusión del arte. Sus instalaciones a partir de distintos lenguajes pretenden alterar la realidad cotidiana de la ciudad, se trata de "la subversión del comportamiento 
humano domesticado por los signos urbanos" en palabras de Milan Ivelic8.

También en este ambiente de obras obligatoriamente efímeras por la realidad económica en la que surgen, encontramos las realizaciones de Ángela Riesco (1947), sólo que aquí el carácter religioso singulariza la producción. Si bien en 1986 recoge la influencia de los trabajos de Catalina Parra de la década de los setenta, dos años más tarde exhibe una instalación denominada Religiosidad Popular, en la que propone una temática de fuerte contenido religioso y popular. Su producción artística se caracteriza por ofrecer una investigación antropológica y sociológica de la cultura popular chilena. Mitos, supersticiones y rituales del universo simbólico nacional se combinan en sus obras.

Carlos Leppe (n.1952) estudió en la Escuela de Bellas Artes de la Universidad de Chile. Su producción se centra en el campo del Arte Conceptual, Performances, Instalaciones y Arte Corporal, utilizando su propio cuerpo como soporte. Mediante estos recursos denuncia la marginación social y cultural: los problemas de la situación artística chilena y el rechazo de la reaccionaria sociedad hegemónica chilena hacia actitudes sexuales políticamente incorrectas. Fue exponente del conceptualismo chileno de mediados de los años 70, corriente conocida como "Escena Avanzada", que propugnaba una ampliación del concepto habitual de arte desde una reflexión crítica.

En esta misma corriente encontramos durante la década de los 70 a Alfredo Jaar $(n .1956)^{9}$. Pese a realizar incursiones en otros ámbitos artísticos serán las instalaciones su principal medio de expresión. Para ello se sirve de todo tipo de elementos des-

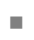

${ }^{8}$ M. IVELiC, Opus. Cit., 1996

${ }^{9}$ Alfredo Jaar: Hágase la luz. Proyecto Ruanda 199498, Barcelona, 1998
}

de fotografías a los proporcionados por los avances tecnológicos, como micrófonos, cámaras de video o monitores de televisión, en un rechazo explícito a la manualidad en el arte. Sus primeros trabajos en Chile incluían al público como espectador activo. A principios de los 80 traslada su residencia a Estados Unidos donde atiende la fractura entre el norte y el sur, entre el desarrollo y el Tercer Mundo. Los basureros nucleares de África o las minas de oro de Brasil son otros de los temas que su producción denuncia. De Jaar dice R.Gutiérrez: "Con fotografías y espejos crea un mundo que nos habla y nos mira"10.

Ese anteponer la idea a la realización material de la obra lo reencontramos en Juan Pablo Langlois (Vicuña (1935 o 36). En 1968 abandona su trabajo como arquitecto para dedicarse a la actividad artística a través de experiencias visuales que exceden la pintura o la escultura, anunciando ya las futuras instalaciones. En 1969 presenta en el Museo Nacional de Bellas Artes de Santiago una polémica instalación denominada Cuerpos blandos. En ella Vicuña exhibe unas bolsas de polietileno rellenas de periódicos y unidas entre sí hasta formar una manga de unos 200 metros que recorría las salas del museo y se prolongaba por una ventana hacia la calle hasta quedar amarrada a una palmera. Durante la década de los 70 utiliza papel de embalar y periódicos para construir figuras humanas, casas, árboles o caballos, en un desmontaje analítico del concepto de escultura. En los años 80 la obra de Vicuña sigue investigando el lenguaje del arte esquivando toda preconcepción.

Otros nombres a considerar son los de Soledad Salame (n.1954) con sus instala-

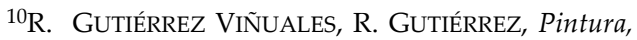
escultura y fotografía en Iberoamérica, siglos XIX y XX, Madrid, 1997. 
ciones reivindicativas de la preservación del medio natural, o Matilde Pérez (n.1920) quien si bien empieza vinculada al neoplasticismo del Grupo Rectángulo pronto abandona esta tendencia para orientarse a la investigación óptica y cinética. Sus obras presentan dos modalidades: una de efectos ópticos sólo aparentes y otra en la que el movimiento es real mediante juegos de luces y motores eléctricos.

Todas estas propuestas no fueron elaboradas como un todo orgánico, sino tal como señala Ivelic ${ }^{11}$, montadas sobre fragmentos. Se pone así de manifiesto, por una parte, el carácter fragmentario del arte chileno construido a partir de recortes de citas internacionales y por otra la desarticulación del cuerpo social y de las instituciones democráticas a causa del régimen militar.

"Al apartarse de los medios tradicionales de hacer arte, estos artistas se vincularon con procedimientos aportados por la neovanguardia internacional: la reactivación de la propuesta de Duchamp, las instalaciones y performances, el empleo del cuerpo o la combinación de interacción multimedia (fotografías, grabado, serigrafía y video). La apropiación de estos procedimientos no fue un gesto mimético ni una actitud sumisa frente a los modelos internacionales, sino más bien un proceso recontextualizador en el propio medio chileno". En todo caso, parece evidente que movimientos como Fluxus no pueden obviarse en una valoración rigurosa.

Con estos nuevos planteamientos estéticos se hace patente para los artistas la realidad de la situación del arte chileno: el carácter dependiente y la insuficiencia del circuito interno para lograr la presencia de obras autóctonas en el mercado internacional. Se produce entonces, tal como señala
Milan Ivelic "la fractura más radical que ha conocido el arte chileno durante el siglo XX".

Este autor destaca además cómo las nuevas promociones artísticas que ahora surgen no conocieron otro sistema político que el régimen militar. Por eso les son ajenos tanto los programas reivindicativos o de denuncia previos, como el sentimiento de culpa por la democracia perdida. Se trata de una generación que irrumpe en la década de los ochenta con figuras como Sammy Benmayor (n.1956), Carlos Maturana Piña conocido como "Bororo" (n.1954), Omar Gatica (n.1956) o Jorge Tacla (n.1958). Se revaloriza ahora la subjetividad, sin buscar justificaciones en planteamientos teóricos, porque se considera la pintura como proceso autosuficiente y como medio de comunicación autónomo y al margen de cuestiones extra-pictóricas.

No quisiera terminar este recorrido sin aludir a la vigencia de estas nuevas formas de hacer arte entre los autores chilenos. En este sentido baste como ejemplo la reciente exposición de Nicanor Parra en la Fundación Telefónica en Madrid, bajo el título Artefactos visuales. ${ }^{12}$ Parra, ingeniero y conocido sobre todo por su actividad poética desarrolla en esta muestra todo su ingenio para expresar mediante lo que denomina artefactos su mundo y el devenir colectivo del pueblo chileno, a través de la dictadura y la estrechez mental de la burguesía de Santiago, al modo en que lo denuncia también el escritor Jorge Edwards. Es en realidad como si Nicanor Parra hubiese creado todo este montaje para resolver de una forma plástica, no exenta de sarcasmo, la redacción de sus memorias. 

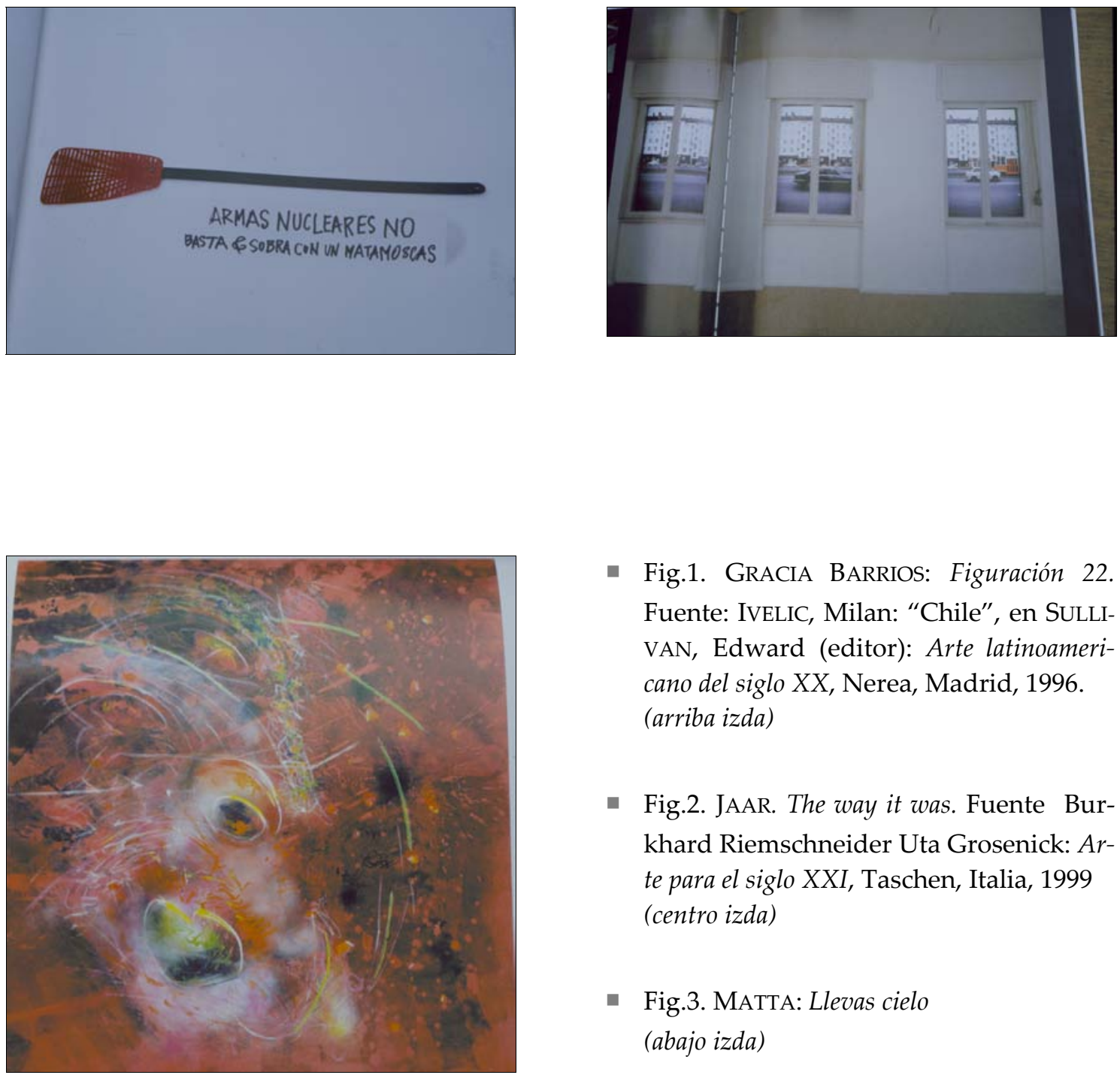

- Fig.1. GRACIA BARRIOS: Figuración 22. Fuente: IVELIC, Milan: "Chile", en SulliVAN, Edward (editor): Arte latinoamericano del siglo XX, Nerea, Madrid, 1996. (arriba izda)

- Fig.2. JAAR. The way it was. Fuente Burkhard Riemschneider Uta Grosenick: Arte para el siglo XXI, Taschen, Italia, 1999 (centro izda)

- Fig.3. MATTA: Llevas cielo (abajo izda)

- Fig.4. PARRA. Artefactos visuales. Fuente VV.AA.: Parra. Artefactos visuales, Madrid, 2001

(arriba dcha)

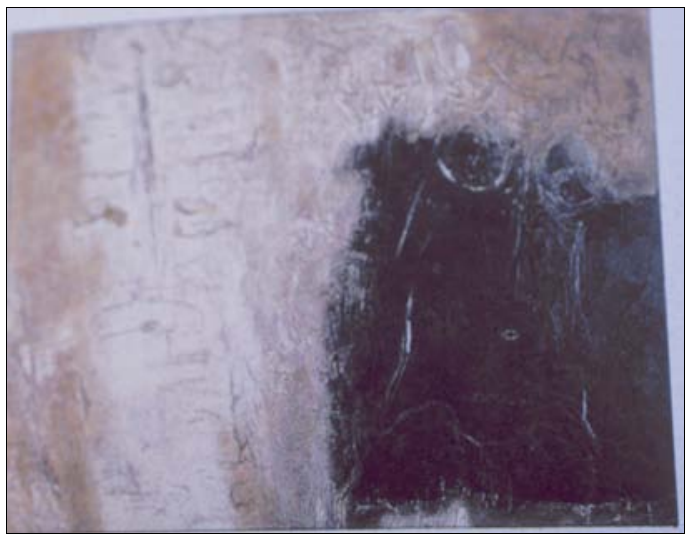

